\title{
SAFETY OF ROAD ROLLER TECHNICAL OPERATION THROUGH DIAGNOSIS
}

doi: $\quad 10.2478 /$ czoto-2019-0043

Date of submission of the article to the Editor: 30/11/2018

Date of acceptance of the article by the Editor: 05/02/2019

\author{
Mikhail V. Doudkin ${ }^{1}$ \\ Adam Idzikowski ${ }^{2}$ - orcid id: 0000-0003-1178-8721 \\ Alina I. Kim ${ }^{1}$ \\ Franciszek W. Przystupa ${ }^{3}$ - orcid id:0000-0002-6849-7151 \\ ${ }^{1}$ East-Kazakhstan State Technical University Kazakhstan \\ ${ }^{2}$ Czestochowa University of Technology Poland, adam.idzikowski@wz.pcz.pl \\ ${ }^{3}$ Wroclaw University of Technology Poland
}

\begin{abstract}
Safety of road roller technical operation for safety diagnosis will be analysed. The analysis is based on the assumptions and hypotheses confirmed by fundamental-applied and experimental-theoretical works. Such modelling enables formulating commonly known diagnostic equivalent for building a real diagnose method of contacting problems of moving roller contacted with the road material.
\end{abstract}

Key words: , diagnosis, modelling, static contact of a roller with a compacted layer, diagnosis

\section{INTRODUCTION}

Errors appear already from the model-based reducing assumptions; they are reinforced through errors of realization, errors of the tools, errors of read-out of measurements, etc. up to errors of interpreting and understanding.

A method of selection of a dedicated diagnoser for a moving roller contacted with the road material in a technical situation was the basic purpose of this scientific work. A trial is made of the use, for diagnosing great working machinery, of standard diagnosing methods being applied in typical machines. The transfer of habits between different systems leads to a reduction of the efficiency of the diagnosing process. In the case of the machine being diagnosed, a model of process, considered to be a standard object of observation and to be indivisible from diagnostic point of view. When the diagnoses for that model has been determined then, for a greater quantity of objects of diagnosis, the method permits to determine the necessary quantity and the quality of simultaneously acting diagnosers. Methods of synthesis of process model for moving roller contacted with the road material have been proposed (Przystupa 2001, Idzikowski et al. 2017).

The considerable complexity and variety of mechanical properties of the contacting bodies (working element of the road roller on the one hand and the deformed soil on the other) have led to the appearance of various schemes for their interaction 
(Ishlinsky 1986, Kaluzhsky and Batrakov 1970, Doudkin et al. 2013). At the same time, analytical solutions on the issue of contact of the movable drum with the material being compacted did not reach a logical result in terms of the possibility of their practical application by design engineers.

The problem of accounting and functional approximation of shearing (tangential) contact pressures, which, along with the normal component, has a significant effect on the coefficient of resistance and stress-strain state of the road surface in the condition of drum rolling (Kim et al. 2017, Surashev et al. 2013) remains acute.

As for the stationary contact, here we have sufficiently effective methods for the evaluation of contact parameters based on Hertz- Belyayev fundamental solution verified experimentally for rollers with circular contour, and also on a physically linear axisymmetric Shtaerman mathematical model for the improved design of a flexible steel drum (Doudkin et al. 2013) with variable geometry of the shell from circular to elliptic profile (Surashev et al. 2013).

\section{DIAGNOSTIC EQUIVALENT}

The diagnostic equivalent for widespread systems is an indicator of the necessity of performing diagnosis, when the object is diagnosed in the form of a multiplied or continuous structure. The basis of the standard is composed of: two shafts, a pair of toothed wheels, four bearings and auxiliary, negligible from diagnostic point of view elements - all these parts being built in a housing. The subassembly consisting of the pair of bearings on the shaft with the toothed wheel is proposed as standard for the equivalent. The load of the whole results from the power being transferred by the assembly. A characteristic feature of that standard is the fact of existence of bearing elements of the shaft as well as of the kinematic pair of higher order in the pair of toothed wheels.

For performing the diagnosis process of a basic assembly at a concrete stage of development of technology, a set of tools and knowledge are necessary. A simple, complete set of tools and of diagnostic knowledge - the DIAGNOSER - requires always time for executing the process of diagnosis. If one technical diagnoser set is at the disposal of the decider, and if one standard basic subassembly is observed, then the time of diagnosis must be shorter than the time between potential damages of the diagnostically observed object. This results from the necessity to be in advance of failures or of an exploitation change of state as a result of the diagnostic decision. Such requirement does not appear in the case of a posterior diagnosis, which identifies the place and the causes of failure.

In the case of an important quantity of objects the quantity of diagnosers functioning at the same time results from the obtained process model.

\section{MODELING OF STATIC CONTACT OF A ROAD ROLLER WITH A COMPATIBLE MATERIAL}

The modeling of the static contact of a roller with a compacted layer is based on the assumptions and hypotheses formulated below, confirmed by fundamental-applied and experimental-theoretical works (Doudkin et al. 2013).

1) A flexible drum with a width $B$ rolls without sliding with a constant linear velocity $\mathrm{V}=$ const along an elastically deformable plane, retaining the elliptical contour of the cylindrical surface (Fig. 1) with semiaxes a and b (Doudkin and Abdeyev 2010, Doudkin et al. 2006). 
2) The layer of soil or pavement having a given strain modulus $E_{k}$, is compacted until the residual displacements cease, and the drum shell is assumed to be absolutely rigid and perfectly smooth.

3) The following external force factors of a static nature (Fig. 1) impact the rolling drive drum at an arbitrary moment of time:

- vertical force $G_{B}$, equal to the mass of the roller and the corresponding part of the frame;

- the required horizontal component or traction load $\mathrm{F}_{\mathrm{T}}$;

- torque moment $\mathrm{M}_{\kappa \ni}$, transmitted by the transmission of the roller to the drum;

- normal $\mathrm{q}_{\mathrm{y}}$ and shearing $\mathrm{q}_{\mathrm{x}}$ reactive distributed loads in the mobile reference system XOY (Fig. 1) along the contacting surfaces of interacting bodies directed parallel to the $Y$ and $X$ axes;

- adhesive force $F_{c \ni}$ of compacted material with drum, which keeps the roller from sliding and applied at point $\mathrm{O}$ (resultant to the pressure $\mathrm{q}_{\mathrm{x}}$ ).

The momentary force $M_{\kappa \ni}$ depends on the power of the $W_{M \ni}$ of the engine of the roller for its movement in the steady-state operating mode at a velocity $V=$ const amount of round per minute $n_{\ni}$. Taking into account the known relationships between the dimensions $(1 \mathrm{~kg}=9,81 \mathrm{H} ; 1$ h.p. $=0,736 \mathrm{~kW} ; 1 \mathrm{~kW}=102 \mathrm{kgm} / \mathrm{s}$ ) (Bronstein and Semendyayev 1962), the following result will be received:

$$
M_{\mathrm{K \ni}}=973,6 \frac{W_{\mathrm{M} \ni}}{n_{\ni}}(\mathrm{kgm})=9550 \frac{W_{\mathrm{M}}}{n_{\ni}}(\mathrm{N} \cdot \mathrm{m}),
$$

where the parameter $n_{\ni}$ is related to the linear velocity $V$ and semi-minor axis $b$ of ellipse.
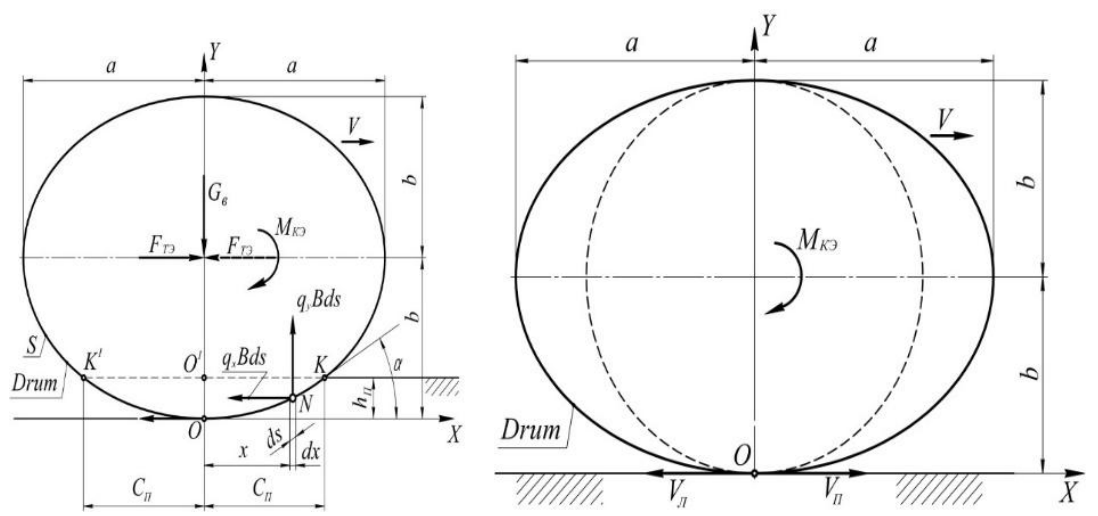

Fig. 1 (left) - Diagram of movable flexible drum contact with a compactable material

Fig. 2 - for the determination of rounds per minute $n_{\ni}$ of the drum

Formula (2) is derived from the solution of the classical kinematics problem on the displacement of a point with a constant angular speed:

$$
\omega_{\ni}=2 \pi n_{\ni}
$$

on an elliptical curve that is the guide of the cylindrical surface of the drum (Fig. 2), moving without sliding with a velocity $\mathrm{V}=$ const (Voronkov 1961, Idzikowski et al. 2017).

When calculating the parameter $\omega_{\ni}$, in the appendix to the scheme shown at Figure 2 , the point $O$ shall be considered, consider the point $O$ at $y=0$. The relative linear 
velocity $\mathrm{V}_{\text {л }}$ of this point is directed to the left along the tangent $\mathrm{x}$ and is expressed by the product (Voronkov 1961, Idzikowski et al. 2017):

$$
V_{\text {Л }}=-b \omega_{\ni}, \quad V_{\Pi}=V
$$

Since, according to the accepted assumption, the drum rolls without sliding, the absolute velocity $\mathrm{V}_{\mathrm{A}}$ of the point $\mathrm{O}$ at a given time is zero, i.e.:

$$
V_{\mathrm{A}}=\left|V_{\Pi}+V_{\text {Л }}\right|=0 .
$$

and therefore, taking into account (3):

$$
b \cdot \omega_{\ni}=V, \quad \omega_{\ni}=\frac{V}{b} .
$$

After elimination of $\omega_{\ni}$ from formulas, we obtain the equating for $n_{\ni}$.

4) The specific pressures $q_{x}=q_{x}(x), q_{y}=q_{y}(x)$, are assumed to be constant along the generatric cylindrical surface of the drum and depending only on the arbitrary distance $x$ to the vertical plane $x=0$, passing through the center of the ellipse (Fig. 1). This premise, gives a basis for schematizing the stress state under consideration in the form of a plane problem of mechanics of a deformable body (Doudkin et al. 2013).

5) When the roller moves, the reactive forces $q_{x}$ and $q_{y}$ from the side of the compacted surface are transferred to the rotating surface of the drum through half of the arc K'OK, i.e., they operate within:

$$
0 \leq \mathrm{X} \leq \mathrm{C}_{\Pi}
$$

Where:

$C_{\Pi}$ - is still the unknown length of the half-chord K'K (in the case of drum rolling).

Here are the formulas for calculating the parameters $\mathrm{C}, \mathrm{h}$, the dependence of the normal pressure $\mathrm{q}_{\mathrm{y}}=\mathrm{q}=\mathrm{q}(\mathrm{x})$ in coordinate system XOY (Fig. 1 ) and its maximum value $\mathrm{q}_{\mathrm{M}}=\mathrm{q}(0)$, taking into account that $\mathrm{q}_{\mathrm{x}}(\mathrm{x})=0$ (Abdeyev et al. 2010, Doudkin and Abdeyev 2010):

$$
\begin{gathered}
q=\frac{q_{\mathrm{M}}}{c} \Phi(x)=\frac{q_{\mathrm{M}}}{c} \sqrt{c^{2}-x^{2}}, \\
q_{M}=\frac{2 G_{\mathrm{B}}}{\pi B C} ;-\mathrm{C} \leq \mathrm{x} \leq \mathrm{C} ; \\
C=a \sqrt{\frac{3,75 \cdot G_{\mathrm{B}}}{\pi \cdot E_{k} \cdot b \cdot B}}=1,0925 a \sqrt{\frac{G_{\mathrm{B}}}{E_{k} b B}}, \mathrm{C} \ll R_{\ni}(0)=\frac{a^{2}}{b} ; \\
h=b\left(1-\sqrt{1-\frac{C^{2}}{a^{2}}}\right) \approx \frac{b^{2}}{2 a^{2}} C^{2}=0,5968 \frac{G_{\mathrm{B}}}{E_{k} B}, h \ll b ;
\end{gathered}
$$

Where:

$$
R_{\ni}(0)=\frac{a^{2}}{b} \text { - is the radius of curvature of the ellipse at the point } \mathrm{O} \text { (Fig. 1); }
$$


$\Phi(x)=\sqrt{c^{2}-x^{2}}-$ is the Hertz-Belyaev-Shtaerman function, which, as noted in, most accurately reflects the physics of the process of interaction between the roller and the compacted material and is comparable to experimental data. With respect to the drum of a circular contour with the radius $R=$ const in equations (9), (10) the changes shall be made (Idzikowski et al. 2017):

$$
R_{\ni}(0)=R, a=b=R
$$

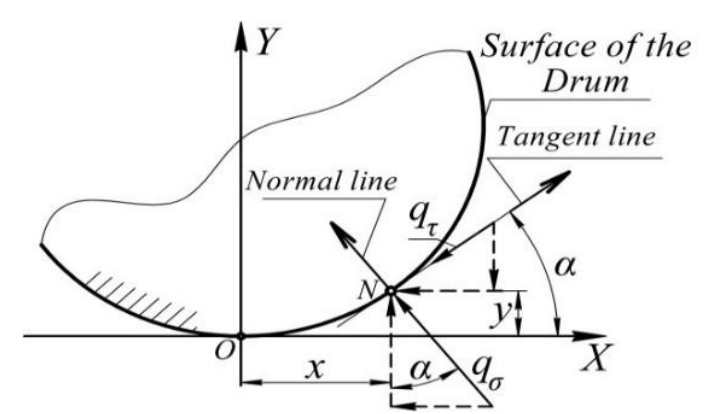

Fig. 3 - Contact stresses (pressures) during drum rolling.

6) In view of the small size of $C, h, C_{\Pi}, h_{\Pi}$, i.e., the slight curvature of the contact surface (see the strengthened inequations (9) - (10), and the analogous ones for $C_{n}, h_{n}$ (Doudkin et al. 2006) and this is proved by investigations, the arc OK is replaced by a chord:

$$
O^{\prime} K=C_{\Pi} \approx O K
$$

7) According to the published papers, we provide a procedure for calculating them using a given radius $R$ of the circular drum surface and eccentricity $\xi$ (Abdeyev et al. 2010):

$$
\xi=\frac{\sqrt{a^{2}-b^{2}}}{a}, \quad 0 \leq \xi<1 ; \text { where for the circle } \xi=0
$$

a) calculation of the length $S$ of the guide line

$$
S=2 \pi R
$$

b) the determination of the relation between $S$ and a through a complete elliptic integral of the $2^{\text {nd }}$ kind $E(\xi)$ in the form of Legendre (Doudkin et al. 2013):

$$
S=4 a \int_{0}^{\frac{\pi}{2}} \sqrt{1-\xi^{2} \sin ^{2} \varphi} d \varphi=4 a E(\xi)
$$

c) the solution of the system of equations (13), (15) with respect to the dimensions of $a$ and $b$ of the ellipse (Fig. 1):

$$
a=\frac{s}{4 E(\xi)}, \quad b=a \sqrt{1-\xi^{2}}
$$




\section{THE CALCULATION OF THE TRACTION LOAD $\boldsymbol{F}_{\mathrm{T} \ni}$ AND THE GENERAL SOLUTION OF THE PROBLEM}

The parameter during the roller movement is the rolling resistance coefficient $f$ (coefficient of rolling resistance), is represented by the semi empirical dependence for circular drums (Kaluzhsky and Batrakov 1970):

$$
f=0,75 \sqrt{\frac{h}{2 R}} .
$$

We modify the formula (17) with respect to a flexible elliptic drum, taking into account inequation (9), assumption 3 ) and replacing, in this regard, $R$ at a small interval $0 \leq X \leq C_{\Pi}$ (Fig.1) y a constant value corresponding to the value of the function $R_{\ni}(x) \mathrm{f}$ the radius of ellipse curvature at the point $O$, when $x=0$ (Fig. 4):

$$
R_{\ni}(0)=\frac{a^{2}}{b}=\text { const }
$$

With replacement of immersion of stationary roller $\mathrm{h}$, and radius $R_{\ni}(0)$, the dependence is:

$$
f_{\ni}=0,375 \frac{b c}{a^{2}}=0,41 \frac{b}{a} \sqrt{\frac{G_{\mathrm{B}}}{b B E_{k}}}
$$

Which in the particular case $a=b=R$, is transformed into a known formula:

$$
f=0,41 \sqrt{\frac{G_{\mathrm{B}}}{R B E_{k}}}
$$

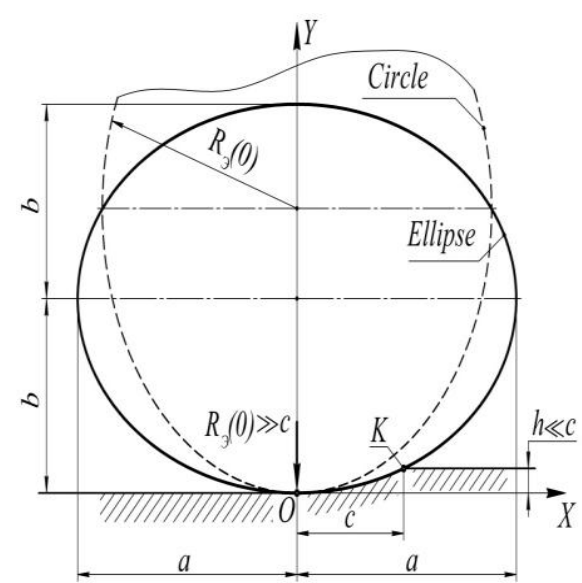

Fig. 4 - Calculation scheme for deriving the formula of the coefficient of resistance $f_{\ni}$

At Figure 5 the functions $f=f\left(E_{k}\right), \quad f_{\ni}=f_{\ni}\left(E_{k}\right)$ and traction loads are provided:

$$
F_{T}=F_{T}\left(E_{k}\right)=f \cdot G_{\mathrm{B}}, \quad F_{\mathrm{T}}=F_{\mathrm{T}}\left(E_{k}\right)=f_{\ni} \cdot G_{\mathrm{B}}
$$


for three specific values $\xi=0$ (circle), $\xi=\frac{\sqrt{2}}{2}=0,7071, \xi=\frac{\sqrt{3}}{2}=0,866$ if $B=1400 \mathrm{~nm}$, $\mathrm{R}=600 \mathrm{~mm}, \quad \mathrm{G}_{\mathrm{B}}=42500 \mathrm{~N} ; \quad \mathrm{E}_{\mathrm{k} 1}=8 \mathrm{mPa} ; \quad \mathrm{E}_{\mathrm{k} 2}=20 \mathrm{mPa} ; \quad \mathrm{E}_{\mathrm{k} 3}=30 \mathrm{mPa} ; \quad \mathrm{E}_{\mathrm{k} 4}=65 \mathrm{mPa}$; $E_{k 5}=116 \mathrm{mPa}$ (Doudkin et al. 2013, Idzikowski et al. 2017).

Guided by these initial data and tables of elliptic integrals (Bronstein and Semendyayev 1962), we have, in accordance with the previous relations:

Case 1 , when $\xi=0$ (circle):

$$
\begin{aligned}
& \left.\begin{array}{c}
a=b=R=600 \mathrm{~mm} ; E(\xi)=E(0)=\frac{\pi}{2} \approx 1,5708 ; \\
S=2 \pi R=2 \cdot 3,1416 \cdot 600=3770 \mathrm{~mm} ;
\end{array}\right\} \\
& f=f\left(E_{k}\right)=0,41 \sqrt{\frac{42500}{600 \cdot 1400 E_{k}}}=\frac{0,0922}{\sqrt{E_{k}}}
\end{aligned}
$$

$\underline{\text { Case } 2}$ - elliptic shell $\left(\xi=\frac{\sqrt{2}}{2}=0,7071\right)$ :

$$
\begin{aligned}
& \left.\begin{array}{r}
S=3770 \mathrm{~mm} ; \quad E(\xi)=E\left(\frac{\sqrt{2}}{2}\right)=1,3506 \\
a=697,8 \mathrm{~mm}, b=493,4 \mathrm{~mm}
\end{array}\right\} \\
& f_{\ni}=f_{\ni}\left(E_{k}\right)=0,41 \cdot \frac{493,4}{697,8} \sqrt{\frac{42500}{493,4 \cdot 1400 E_{k}}}=\frac{0,0719}{\sqrt{E_{k}}} ;
\end{aligned}
$$

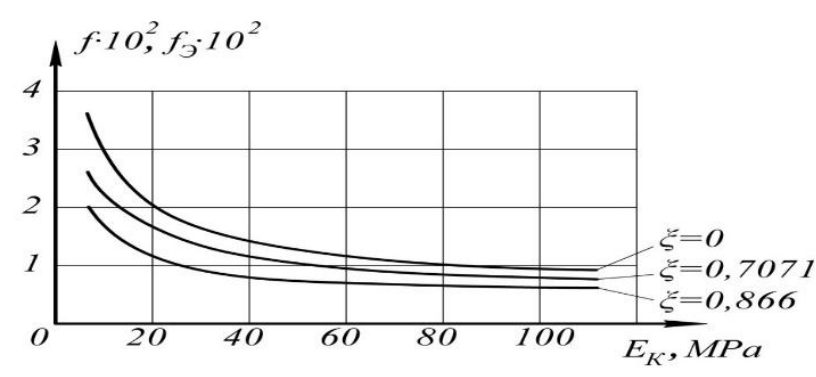

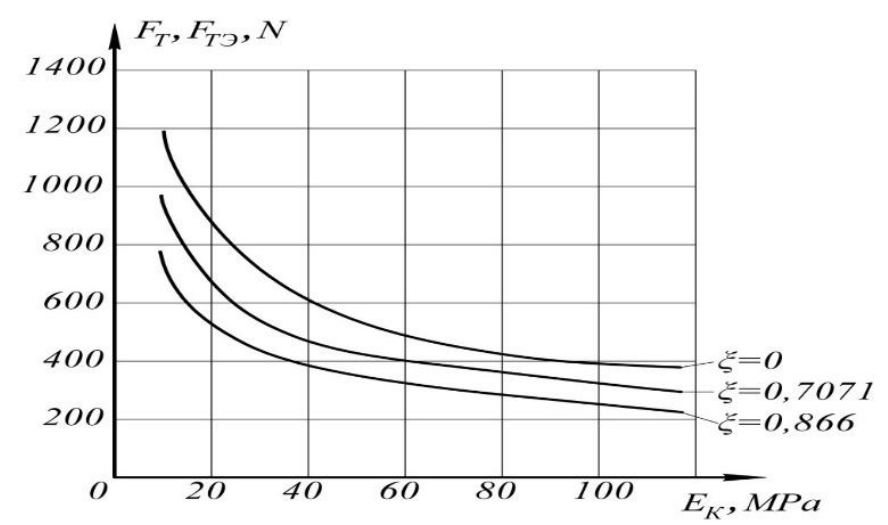

Fig. 5 - Dependence diagram for $f\left(E_{k}\right), f_{\ni}\left(E_{k}\right), F_{T}\left(E_{k}\right), F_{\mathrm{T}}\left(E_{k}\right)$

Comparing the similarly-named curves at figure 5 we can note that with an increase in the degree of ovalization of the roller shell, that is, with an increase in the parameter $\xi$, characterizing the elongation of the ellipse in the direction of axis $\times$ (Fig. 1), the resistance coefficient $f_{\ni}$ and traction force $F_{\mathrm{T}}$ is decreased compared with the analogous values of $f$ and $F_{T}$ for a drum with a circular contour. In the examples of 
calculation considered this effect corresponded to $21.7 \%$ with $\xi=\frac{\sqrt{2}}{2}=0,7071$ and 38 $\%$, when $\xi=\frac{\sqrt{3}}{2}=0,866$.

Continuing the mathematical description of the multifunctional complex contact problem of applied mechanics, we approximate the pressures $q_{y}, q_{x}$ by the next analysis.

Generalizing the symmetric physical-mathematical model (Abdeyev et al. 2010) for the drum rolling process and taking into account the simplifications, we provide the equilibrium equations (see Figure 1), taking into account the presented analytical relations.

In this contact problem, it is necessary to find $A_{1}$ and $A_{2}$ of the pressure function. The second constant $A_{2}$ is calculated from equation which we disclose. Such modelling which was shown only in small part enables formulating commonly known diagnostic equivalent for building of real diagnose of contacting problems of moving roller contact with the compacted material.

\section{DIAGNOSTIC EQUIVALENT RD}

The Diagnostic Equivalent for machine components is an index of the need of diagnosing realization when the object being diagnosed is an object in the form of a multiplied (continuous) structure. As a basis for a standard of that index a basic assembly in machines is proposed - a single-stage gear transmission. That gear is composed of two shafts, a pair of toothed wheels, four bearings and auxiliary elements that are negligible from the diagnosing point of view - the whole built in a body. As standard for the index the following subassembly is proposed: a pair of bearings on a shaft with a toothed wheel. The load of the whole results from the power being transferred by the assembly. A characteristic feature of that standard is the existence of bearing elements of the shaft and a kinematic pair of higher order in the pair of toothed wheels. "Standard" for the diagnostic inde is a pair of bearings on a shaft with a toothed wheel.

For the realization of the diagnosing process of that basic subassembly - the pair of bearings on the shaft with a toothed wheel - a set of tools and of knowledge is necessary on a concrete stage of technology progress. The set of tools and of knowledge - the diagnoser - requires a certain time for the realization of the process of diagnosis. If the decider disposes of one technical set of the Diagnoser and if one basic standard subassembly is observed, then the time of diagnosis must be shorter than the period of time between potential damages of the diagnostically observed object. This results from the necessity to anticipate a failure by a diagnostic decision. Such requirement does not appear in the case of a posteriori diagnosis that identifies the place and cause of the failure.

In the case of objects being diagnosed, it is the diagnostic equivalent that will be an important facilitation - a subassembly of a pair of rolling bearings on a shaft with a toothed wheel. When the indispensable frequency of purposeful diagnoses Fd for the standard elementary subassembly has been determined then, in the case of a greater quantity of objects being diagnosed, the diagnostic equivalent permits to determine the necessary quantity of simultaneously functioning diagnosers.

The quantity of simultaneously functioning diagnosers results from the necessary frequency of diagnoses $F d$ being repeated on a concrete assembly. The analysis of 
the frequency of diagnoses results from the goal of diagnosing, the reliability characteristics and economic characteristics of transporting (Przystupa 2007).

For example - The quantity of diagnostic equivalents will be as follows:

- a pair of bearings on a shaft with a toothed wheel - 1

- a single-stage transmission gear - 2, a two-stage transmission gear - 3a roller -1 , a set of 6 rollers -6 ,

- conveyor - some scores of thousands, conveyor system - several hundreds of thousands.

Notwithstanding the so important quantitative differences, measurable through the quantity of diagnostic equivalents, the diagnosticians transfer diagnostic methods suitable for elementary objects to objects with a value of $\mathrm{Rd}$ that is several orders greater. By laboratory methods one can diagnose a single object even in the work field; that can be, however, performed in extraordinary situations (failures, construction of the space of diagnostic parameters, etc.). Another situation appears in the case of mass objects but used as single objects (automotive vehicles) when the user is ready to cover important costs for assuring the security level. On the basis of Author's own experience, the following diagnosing methods can be proposed for the Quantity of diagnostic equivalents Rd (Przystupa 2007):

$\mathrm{Rd}<10$ - Single tools, laboratory instruments, scientific instruments

$10<\mathrm{Rd}<100$ - Single tools for the object, inspecting instruments - here is the Rd for modelled roller

$100<R d<1000$ - Local multi-task tools for analogical objects with important

quantities, testers, inspection instruments, elementary instruments

$\mathrm{Rd}>1000$ - Testers with possibility of displacement.

Technical diagnosing methods for a road roller can be limited to methods that are economically acceptable:

- effort methods, methods of direct observation

- efficiency methods, VBA, thermographic methods, temperature methods

- wear methods - solid materials, oils, geometric methods

- electromagnetic methods, electro-inductive-capacitive-resistant methods, etc.

- ultrasound methods, X-ray methods, etc., marker methods - visual, radiographic methods.

\section{CONCLUSIONS}

The above mathematical analysis allowed to formulate the following conclusions:

1) The presented process mechanical-mathematical model is fully comparable to the conceptual hypothesis of Kharhuta-Babkov (confirmed experimentally) about the pressure $q_{y}$ distribution over the surface of moving roller contact with the compacted material and the classical preconditions for the mechanics of an elastically deformable body as applied to the process of contact interaction between a drum and a road surface.

2) A new analytical dependence is obtained for the rolling resistance coefficient $f_{\ni}$, which generalizes the known formula (22) for the driving and driven drums having an elliptical contour of the perimeter $S$ of the shell.

3) It is proved that, in the case of the roller's movement, the width $C_{\Pi}$ of the contact surface decreases, and this fundamental fact agrees with the fundamental provisions (contradicting the existing applied calculation schemes, where 
$C_{\Pi}=C$ ), and the maximum contact pressure $q_{\mathrm{мy}}$ increases with increasing degree of drum ellipticity, characterized by eccentricity $\xi$ and coefficient $\zeta$ $(0 \leq \xi<1,0<\zeta \leq 1)$.

4) The developed calculation theory makes it possible to optimize all the main parameters of the contact interaction of a rotating drum with a compaction layer by adjusting the design dimensions of semi-axes $a, b$ of the elliptical surface of a flexible cylindrical shell with a smoothly variable geometry, thereby increasing the quality of the constructed road or new ground base. The best sealing effect with an intensive accumulation of residual deformations is achieved when qмy $=(0,8 \div 0,9)$ бпр.

5) Such modelling enables formulating commonly known diagnostic equivalent $\mathrm{Rd}$ for building of real diagnose method of contacting problems of moving roller contact with the compacted material. The Diagnostic Equivalent for machine components is an index of the need of diagnosing realization when the object being diagnosed is an object in the form of a multiplied (continuous) structure.

\section{REFERENCES}

Abdeyev B.M., Dudkin M.V., Rechitsky S.V. 2010. Mathematical modeling of the interaction of a stationary flexible drum of a road roller and compacted material // Nanomaterials for the protection of industrial and sub-terrestrial structures: Materials of the international Symposium and XI International Conf. "Solid State Physics", June 9-12, 2010. - Ust-Kamenogorsk: EKSTU n.a. D. Serikbayev, p. 8187

Bronstein I.N., Semendyayev K.A. 1962. Guide book on mathematics for engineers and students of technical colleges. - Moscow: State Publishing House of Physical and Mathematical Literature, Moscow.

Doudkin M.V., Pichugin S.Yu., Fadeev S.N. 2013. Contact Force Calculation of the Machine Operational Point. Life Science Journal - 2013. - Vol. 10. - Issue 10. Article number 39 - P. 246-250. SJR_2013:0,139 (Scopus).

Doudkin M.V. Pichugin, S.Yu., Fadeev. S.N. 2013. The Analysis of Road Machine Working Elements Parameters. World Applied Sciences Journal -2013. - Vol. 23. - Issue 2 - P. 151-158. SJR_2013:0,222 (Scopus).

Doudkin M.V., Pichugin S.Yu., Fadeev S.N. Vavilov S.N. 2013. Calculation of the Interaction of Working Body of Road Machine with the Surface. Life Science Journal - 2013. Vol. 10. - Issue 12. Article number 133. - P. 832-837. SJR_2013:0,139 (Scopus).

Doudkin M.V., Pichugin S.Yu., Fadeev. S.N. 2013. Studying the Machines for Road Maintenance. Life Science Journal - 2013. - Vol. 10. - Issue 12. Article number 24 - P. 134-138. SJR_2013:0,139 (Scopus).

Doudkin M.V., Abdeyev B.M. 2010. Investigation of the interaction between a stationary flexible drum of a road roller and compacted material / Jubilee collection dedicated to the 75th anniversary of the birth of the first head of the department "Construction and Road Machines", Doctor of Technical Sciences, Professor Yantsen Ya.A. - Karaganda: "Bolashak-baspa". p. 99 - 104.

Doudkin M.V., Kuznetsov P.S., Sakimov M.A., Golovin A.A., Kiyalbayev A.K. 2006. Road roller drum. Preliminary patent of the Republic of Kazakhstan 18131. 
Certification agency of the Republic of Kazakhstan No. 51084. IPC E01C 19/26. Published Statement No. 12; 15.12.2006.

Idzikowski A., Doudkin M.V. Fröhilich S.R. i inni 2017. Modelling of machinery processes, Monograph, vol. II, Publishing Office of Faculty of Menagement Czestochowa University of Technology, Czestochowa

Idzikowski A., Doudkin M.V. Przystupa F.W. i inni 2017. Modelling of machinery processes, Monograph, vol. I, Publishing Office of Faculty of Menagement Czestochowa University of Technology, Czestochowa

Ishlinsky A.Yu. 1986. Applied problems of mechanics. Book 1. Mechanics of viscoplastic and not completely elastic bodies. - Moscow.

Kaluzhsky Ya.A., Batrakov O.T. 1970. Sealing of road base and pavement layers. Publishing house "Transport", Moscow

Kim A., Doudkin M.V., Vavilov, Guryanov G. 2017. New vibroscreen with additional feed elements. Archives of Civil and Mechanical Engineering. Volume 17, Issue 4, September 2017, Pages 786-794. https://doi.org/10.1016/j.acme.2017.02.009.

Permyakov V.B. Justification of the choice of the parameters of vibrating rollers / V.B. Permyakov, A.V. Zakharenko, S.V. Savelyev / Higher educational institutions oublishing house "Construction". - 2003. - No. 2. - p. 100-103.

Permyakov V.B. Perfection of the theory, calculation methods and design of machines for compaction of asphalt-concrete mixtures: Doctoral thesis: 05.05.04. - St. Petersburg, 1992. - 412 p.: ill.

Przystupa F.W. 2007. Diagnostics of "UUUU..." type situations in Logistic Systems. Systems (Wrocław), vol. 12, nr 2, p. 21-28,

Przystupa, F.W. 2001. Diagnostics of a wide area object; case of systems of belt conveyors, SYSTEMS, Vol. 6/1-2, 2001, pp. 135-157, Oficyna Wydawnicza Politechniki Wrocławskiej, Wroclaw.

Sergeeva T.N. Choice of roller parameters for compaction of asphalt-concrete coatings: author's abstract from Doctoral Thesis / T.N. Sergeeva.-L., 1981.

Surashev N., Doudkin M.V., Yelemes D, Kalieva A. 2013. The Planetary Vibroexciter with Elliptic Inner Race. Advanced Materials Research Vols. 694-697 (2013), pp 229-232. Trans Publications, Switzerland, doi: 10.4028/ AMR.694-697/229.

Voronkov I.M. 1961. Course of theoretical mechanics. Textbook for students of higher technical educational institutions. Moscow: State Publishing House of Physical and Mathematical Literature.

Zubkov, A.F. 2008. Development of technology for the installation of asphalt-concrete coatings with increased operational parameters. Doctoral thesis. - Voronezh, VGASU. 